\title{
BMJ Open Living conditions, HIV and gender affirmation care pathways of transgender people living with HIV in France: a nationwide, comprehensive, cross-sectional, community-based research protocol (ANRS Trans\&HIV)
}

Marion Mora (D , , ${ }^{1,2}$ Giovanna Rincon, ${ }^{3}$ Michel Bourrelly, ${ }^{1,2}$ Gwenaëlle Maradan, ${ }^{1,2}$ Anaenza Freire Maresca, ${ }^{4}$ Florence Michard ${ }^{5}$ Elisabeth Rouveix, ${ }^{4}$ Julie Pannetier, ${ }^{6}$ Diane Leriche, ${ }^{7}$ Tristan Alain, ${ }^{8,9}$ Yazdan Yazdanpanah, ${ }^{5}$ David Michels, ${ }^{8,9}$ Bruno Spire ${ }^{1,2}$

To cite: Mora M, Rincon G, Bourrelly $\mathrm{M}$, et al. Living conditions, HIV and gender affirmation care pathways of transgender people living with HIV in France: a nationwide, comprehensive, cross-sectional, communitybased research protocol (ANRS Trans\&HIV). BMJ Open 2021;11:e052691. doi:10.1136/ bmjopen-2021-052691

- Prepublication history and additional supplemental material for this paper are available online. To view these files, please visit the journal online (http://dx.doi.org/10.1136/ bmjopen-2021-052691)

Received 05 May 2021 Accepted 22 November 2021

Check for updates

(C) Author(s) (or their employer(s)) 2021. Re-use permitted under CC BY-NC. No commercial re-use. See rights and permissions. Published by BMJ.

For numbered affiliations see end of article.

Correspondence to

Marion Mora;

marion.mora@inserm.fr

\section{ABSTRACT}

Introduction Transgender identity is poorly accepted in France, and data on living conditions and the daily difficulties transgender people encounter are scarce. This lack of data reinforces their invisibility in social life, contributes to their stigmatisation and probably increases the burden of HIV infection, especially for HIV-positive transgender people (TRHIV). The main objective of the community-based research study ANRS Trans\&HIV is to identify personal and social situations of vulnerability in TRHIV, the obstacles they encounter in terms of access to and retention in medical care, and their gender affirmation and HIV care needs.

Methods and analysis ANRS Trans\&HIV is a national, comprehensive, cross-sectional survey of all TRHIV currently being followed in HIV care units in France. TRHIV women are exclusively included in the quantitative component, and TRHIV men in the qualitative component. Data are collected by community-based interviewers and will be analysed to explore patient care pathways and living conditions in the TRHIV population with regard to gender affirmation and HIV. Data collection began in October 2020 and should be completed in December 2021. The statistical analyses techniques used will be adapted to each of the study's objectives and to the type of data collected (cross-sectional (questionnaires) and retrospective (biographical trajectory)). The study's results will provide a greater understanding of TRHIV health needs in order to suggest possible national recommendations for comprehensive HIV and gender affirmation medical care.

Ethics and dissemination ANRS Trans\&HIV was approved by Inserm's Ethical Evaluation Committee (no 20-694 on 12 May 2020) and is registered with the National Commission on Informatics and Liberty under number 2518030720. Potential participants are informed about the study through an information note provided by their attending HIV physician. All results published in peer-reviewed journals will be disseminated to the HIV
Strengths and limitations of this study

The main strength of ANRS Trans\&HIV is the collection of biographical and sociobehavioural data for the first time in France from HIV-positive transgender people (TRHIV) followed in hospital-based HIV care units.

- The involvement of community-based interviewers fosters participants' trust and limits the risk of judgement and discrimination.

One of the study's limitations is that some TRHIV will probably be missed, because of refusal to participate, because they are followed in primary care (ie, non-hospital contexts), and because HIV care units may not identify potentially eligible patients.

transgender community, institutional stakeholders and healthcare providers.

Trial registration number NCT04849767.

\section{INTRODUCTION}

It is difficult to estimate the number of transgender people worldwide, as in most demographic surveys, gender-related data are summarised using a 'man versus woman' distinction. This invisibility is reinforced by several forms of discrimination against transgender people, and, in certain countries, by their criminalisation. ${ }^{1}$

The data collected for gender-affirmative surgery vastly underestimate the true number of people concerned, as surgery is not systematic for economic reasons (expensive and not always reimbursed), ${ }^{2}$ and because some people wish to live their gender without it. ${ }^{3}$ That is why, in the present study, transgender 
refers to all persons whose self-identified gender is different from the sex they were assigned at birth. This is the same definition used in the 2009-2010 Canadian community-based research study Trans Pulse. ${ }^{4}$

Trans Pulse explored the experiences and social determinants of transgender people's health. It identified employment discrimination, ${ }^{5}$ discrimination in healthcare services $^{6}$ and a higher suicide rate ${ }^{7}$ in this population. Higher rates of suicidal behaviour have also been described in other transgender contexts ${ }^{89}$ and are associated with discrimination and family rejection ${ }^{10}$. Trans Pulse also showed that racial/ethnic and gender discrimination can increase HIV infection risk in transgender people. ${ }^{11}$ A systematic review, covering January 2006 to March 2017, highlighted gender disparities between transgender men and transgender women in terms of HIV infection risk and risky sexual practices. ${ }^{12}$ In the USA, HIV prevalence in transgender women is high, especially for African-American and Latina women. ${ }^{13}$ In a study of 3818 people living with HIV (PLHIV) in San Francisco, 35 were HIV-positive transgender (TRHIV) women on antiretroviral treatment (ART). Results showed that with respect to non-transgender people, they had a lower rate of adherence to treatment, experienced more side effects, had a higher rate of depression and had less positive interactions with care providers. ${ }^{14}$

Data on TRHIV people are scarce and most only concern TRHIV women. A meta-analysis of 39 studies in 15 countries in 2013 showed an HIV prevalence of $19 \%$ in transgender women, and that the risk of infection was 50 times higher in this population than in the general population. ${ }^{15}$ A systematic review performed between 2012 and 2015 showed that globally, transgender women had a greater risk of HIV infection, the prevalence reaching $40 \%{ }^{16}$ That review also described the association of 'syndemic' factors with the risk of infection. More specifically, some transgender people are exposed to biological and social factors that most likely impact not only HIV infection risk, but also prevention behaviours and disease progression. These vulnerability factors influence ART adherence and viral load control in transgender people who are screened, treated and followed. ${ }^{17} 18$

TRHIV women may also have a greater risk of drugdrug interactions between ART and feminising hormonal regimens. Hormonal treatments may increase the risk of comorbidities (osteopenia, cardiovascular risk factor, venous thromboembolism). However, data on possible interactions are scarce ${ }^{19}$ and contradictory. ${ }^{20}$ TRHIV women are more adherent to ART when they have few side effects and when female hormone effectiveness is not affected. $^{21}$

With regard to transgender men, little information is available about interactions between masculinising hormone and ART. The few studies to date estimating HIV prevalence in this population reported a small number of positive cases, ${ }^{22} 23$ which suggests that the HIV burden is lower in transgender men than in transgender women. TransPulse (see above) is one of these studies; it looked at the effects of testosterone in transgender men who have sex with men and showed that using the hormone did not influence HIV-related sexual risk behaviours, despite the fact that testosterone increases libido. ${ }^{24}$

In France, gender identity is still a complex issue from a legal perspective. The first small step forward towards recognising this population was taken in 2010 with the decree no 2010-125, whereby 'transsexualism' could no longer be considered a mental pathology in the country's social security's system's classification of long-term illnesses. The ' $21^{\text {st }}$ century justice law' ${ }^{25}$ subsequently stipulated that a person can change their sex designation in their civil status if desired, that such a change must not be subject to medical treatment obligations, and that it must be legally recognised.

Despite this progress, many health and social dimensions of transgender people's lives, as well as their precise number in France, remain unknown. In its 2009 report, the French National Authority for Health estimated that between 1 in 10000 to 50000 people were transgender (ie, between 6600 and 33000 transgender people in the general population). ${ }^{26}$ Other estimates were made based on health insurance-based data for requests for genderaffirmative surgery. However, these excluded all persons who do not have surgery, and those who have surgery outside France.

In 2010, the ethnographic, anonymous survey 'Transgender and Sexual Health' aimed to identify and describe the sociodemographic characteristics of transgender people, their patient care pathways regarding their gender affirmation process, their sexual health, and their situation in terms of HIV/AIDS. ${ }^{27}$ Results highlighted difficulties accessing care during gender affirmation. No man and $6.9 \%$ of women declared being HIV positive. The HIV prevalence rate was higher among women who were sex workers (SW) (17.2\%), especially SW born outside of France $(36.4 \%){ }^{28}$ Furthermore, between 2012 and 2016, Santé Publique France—the national public health agency-recorded 123 TRHIV (110 TRHIV women, 11 TRHIV men, and 2 unspecified). The majority resided in the Île-de-France region (66\%) and came from the Americas $(75 \%)$. Only 13\% were born in France.

Transgender people are more affected by intersectional stigma, ${ }^{29}$ specifically gender identity discrimination, combined with stigma related to HIV, sex work and migration. In 2007, an exploratory study exploring transgender people's social situation, sexual behaviours and use of healthcare, showed they were more socially isolated than the general population, that one in three reported discrimination in getting employment, that 1 in 5 had decided not to seek healthcare care for fear of discrimination, and that they took significant risks in terms of HIV infection exposure. ${ }^{30}$

PLHIV are still subject to multiple forms of discrimination which hinder them from achieving their 'life project' and can compromise therapeutic success. For example, the ANRS-VESPA2 survey, conducted in 2011 showed that PLHIV still experienced discrimination in employment 
$(24 \%)$, in their family $(11 \%)$, and in healthcare services $(8 \%){ }^{3132}$

Although sex work was legalised in France in 2016, the law penalises clients; this is detrimental to SW safety, health and living conditions (eg, more risks at work, less condom use). ${ }^{33}$ These negative effects are more frequent in transgender SW. ${ }^{34}$

In France, universal healthcare covers all public medical costs for people working or residing in the country on a stable basis, and PLHIV receive free healthcare. Access to care is more difficult for PLHIV whose administrative situation is irregular (eg, no work permit). Migrant people or people with social vulnerability respond less well to ART. ${ }^{35}$

A survey analysing the Bichat hospital's HIV care unit database in 2015 showed that transgender people were more exposed to HIV and other sexually transmitted infections (STI) than other populations, and that their dermatological complications needed better management. ${ }^{36} \mathrm{~A}$ second survey in the same care unit, which aimed to highlight the dangers associated with the clandestine use of cosmetic surgery, reinforced these results and showed that transgender women also presented physical health risks related to the illicit use of silicone. ${ }^{37}$

In order to improve knowledge about the situation of TRHIV in France, we designed the community-based research study ANRS Trans \& VIH, which aims to better understand this population's living conditions and healthcare pathways. To encourage TRHIV to participate, we partnered with the transgender self-support association ACCEPTESS-T, and AIDES, a long-established international association in the fight against HIV. Both associations have in-depth knowledge of the issues and problems facing TRHIV. Numerous epidemiological studies have shown the value of involving associations in research for a better understanding of community-based health problems, ${ }^{38}$ especially in the most marginalised populations. ${ }^{39}$

Both associations were fully involved in the conception and writing of the study protocol, the coconstruction of the research questions and data collection tools. They highlighted important issues to be investigated (gender affirmation trajectories and specific discrimination situations), played a role in adapting the questionnaire and interview guide, and suggested how the field survey could be organised. They are also fully implicated in the ongoing data collection process.

\section{OBJECTIVES}

The main objective of ANRS Trans\&HIV is to identify personal and social situations of vulnerability in TRHIV, the obstacles they encounter in terms of access to and retention in medical care, and their gender affirmation and HIV care needs.

\section{Specific objectives}

1. Describe the life trajectories of TRHIV, especially life events which may represent HIV vulnerability factors.
2. Document access to and retention in HIV care by estimating the burden of social and psychosocial factors, as well as experiences of discrimination and perceived stigma.

3. Document sexual health (ie, sexuality according to TRHIV transition trajectory, risk taking (sexual or related to substance use)), and its relationship to prevention; and establish these factors' impact on access to and retention in HIV care. Document the impact of the ongoing COVID-19 health crisis on everyday TRHIV experience.

4. Identify the specific needs and health of TRHIV men.

\section{METHODS AND ANALYSIS \\ Study design}

ANRS Trans\&HIV is a national, comprehensive, crosssectional community-based research study of TRHIV followed in hospital-based HIV care units in France. By 'comprehensive', we mean that all TRHIV women and men frequenting these HIV care units will be invited to participate. To estimate the study sample size for ANRS Trans \& HIV, we conducted an exploratory survey in 258 HIV care units in 2018. Of these, 53 had at least one TRHIV in their active patient file, for a total of -890 TRHIV women and 5 TRHIV men. Given the small size of the active patient file, we decided to conduct a comprehensive survey instead of a sampling-based one. Recruitment is still ongoing and we hope to have similar numbers of TRHIV (ie, 890 and 5) in the present study.

ANRS Trans\&HIV uses two approaches to explore TRHIV life trajectories and healthcare pathways, as well as their living conditions with regard to gender affirmation and HIV. The first approach is quantitative, where data are collected to measure the difficulties encountered by TRHIV women, in order to inform public policy. The second approach is qualitative, whereby data are collected for TRHIV men to help describe their needs and living conditions. Data collection began in October 2020 and is should be completed in December 2021. Dissemination of results will likely start in late 2022.

\section{Study procedure}

All physicians of participating HIV care units will invite all their TRHIV to participate in the study. The study protocol specifies that they offer the survey to all TRHIV in their active patient file. TRHIV are invited to participate by their attending HIV doctor at a planned medical visit. The doctor presents the study, its objectives, benefits and constraints, and answers any questions the TRHIV have. The doctor indicates that participation is voluntary, and that the potential participant has the right to withdraw at any time without justification and without any consequence on the quality of the care received. The doctor also provides the TRHIV with an information note for personal reading.

The study's interviewers come from the transgender community, and are trained in techniques in 
administering questionnaires. They were recruited based on their proficiency of French, Spanish and Portuguese, which are languages mainly spoken by the population concerned. The decision to recruit transgender interviewers was made to foster participants' trust and limit the risk of judgement and discrimination.

- Transgender women who agree to participate in ANRS Trans\&HIV take part in the quantitative component only. They are referred to an interviewer in a dedicated room, so that the associated sociodemographic and life-event questionnaires can be administered privately to them.

- As there are so few transgender men those who agree to participate are involved in the qualitative component only. Qualitative interviews are conducted privately by an interviewer (researcher) in a dedicated room. Interviews are recorded only with participants' consent.

People who refuse to participate are asked by their attending HIV doctor to complete a short questionnaire to collect the reasons for their refusal as well as sociodemographic characteristics, in order that any biases due to non-responders can be evaluated later in the analyses.

\section{Quantitative data collection}

The quantitative component collects sociobehavioural and medical information on TRHIV women using three questionnaires (sociodemographic, life event and medical). Questionnaires are administered face to face by an interviewer.

Different questionnaire modules provide information on different aspects of participants' lives: sociodemographic characteristics; life conditions (employment, financial resources, housing); HIV testing and management; drug use; social relations; gender affirmation trajectory; self-esteem; mental health; sex life. Discrimination is measured using a scale adapted from The Trajectories and Origins survey ${ }^{40}$ which explored discrimination in various contexts including employment, family, services, healthcare, ethnic origin, trans identity, HIV status and dress code. The impact of the ongoing COVID-19 health crisis and France's two lockdowns on participants is measured at the financial (employment and available resources), medical (impact on healthcare) and relational levels. The face-to-face questionnaire, in French, is provided as a online supplemental file.

Community partners from ACCEPTESS-T and AIDES were involved in adapting the questionnaires and interview guide to the study population. For example, in the gender affirming trajectory section in the questionnaire, they suggested questions such as 'When did you first identify yourself as a woman?' and 'By what means?' with 'Makeup, Wig/long hair, Removable prostheses, Clothing, shoes (dresses, skirts, heels, etc.), Hair removal, and Other' as response options. It was very important for the community that this question be asked so that researchers could discover whether there is a specific moment and a specific way in the lives of transgender people where they self-identify as women, or whether it is a progressive process.

The life-event questionnaire is based on that used in the ANRS Parcours survey. ${ }^{41}$ It makes it possible to retrospectively reconstruct the life trajectory of TRHIV women for certain factors that may have impacted (1) their becoming infected with HIV, (2) their healthcare situation, and, more generally (3) their current life. Furthermore, it makes it possible to retrace their migratory, residential, administrative and gender affirmation trajectories as well as their healthcare pathways. The life-event questionnaire, in French, is provided as a online supplemental file.

The medical questionnaire collects data from various medical records (nadir CD4, HIV viral Load and ART therapies, contamination mode, gender affirmation therapy/surgery, hormone therapy, comorbidities, osteoporosis, pathologies related to problems related to physical changes; mental health history; STI and other co-infections). The medical questionnaire, in French, is provided as online supplemental file.

All these data will make it possible to create an inventory of the state of health of the TRHIV women surveyed, which can then be compared with the state of health of the general population of PLHIV.

\section{Qualitative data collection}

The qualitative component with TRHIV men involves a faceto-face individual interview with a researcher. Medical data are collected with the same medical questionnaire used in the quantitative component (see above). An interview checklist ensures structure. The opening question is "Starting an identity transition is an important moment in one's life. Could you tell me about your personal experience?" Questions focus on living conditions ( "What can you say about your current living conditions (employment, housing, etc.)?"), migratory trajectory ("In what context did you arrive in France?"), gender affirmation ("How have you managed to affirm and make your gender identity visible?"), HIV acquisition ("When did you learn of your seropositivity?"), and medical follow-up ("Today, can you say that you are satisfied with your medical care?"), as well as the impact of the current COVID-19 health crisis ("How have you experienced the COVID-19 crisis?"). The interview grid, in French, is provided as online supplemental file.

These interviews provide an insight into the practices and experiences of TRHIV men, who constitute a minority HIV population.

\section{Data collection in HIV care units}

To document the healthcare provided to TRHIV another questionnaire collects structural data on the various HIV care units participating in ANRS Trans\&HIV, including the number of doctors, opening hours, specificity of the consultation (therapeutic education or not), the care services offered (eg, psychiatry, endocrinology, proctology), permanent presence of transgender association, etc. These data will be used to construct variables for each 
unit and for the quality of care offered. They will also be used in statistical analyses to identify the potential impact of structural factors on individual factors. The HIV care unit's questionnaire, in French, is provided as a online supplemental file.

\section{Patient and public involvement}

ANRS Trans\&HIV is grounded in community-based participatory research. Transgender community members and representatives of the PLHIV community have been involved in all steps of the study to date: conception of the research question, enrolment and data collection. They will also be involved at the interpretation of the results.

All the results of the ANRS Trans\&VIH study who will published in peer-reviewed journals will be disseminated to the HIV transgender's community, institutional stakeholders and healthcare providers. We will use adapted materials, general public leaflets and articles in Frenchspeaking journals for this. Patients' participation in the study is voluntary, and they have the right to withdraw from the study at any time without justification and without consequence for the quality of care received. To thank them for their time, they are compensated with a twenty-euro gift voucher.

\section{Analyses and expected results}

The statistical analysis techniques will be adapted to each of the study's objectives and the type of data collected (cross-sectional (questionnaires) and retrospective (biographical trajectory)).

\section{Life trajectories of transgender women which may represent} factors of HIV infection vulnerability:

The demographic and socioeconomic characteristics of TRHIV women participating in the quantitative component will first be described. The data collected in the lifeevent questionnaire will make it possible to study the link between life trajectory and HIV infection risk ${ }^{42}$ in general in transgender women for various contexts (residential, administrative, sexual and emotional, gender transition stage, etc) that expose them to the risk of HIV infection, and other contexts that facilitate or hamper general and HIV-specific healthcare in those who become infected. These data will also help us to better understand the current living conditions and health needs of TRHIV women, and will be analysed with techniques adapted to retrospective data (eg, group-based trajectory model technique) in order to identify specific profiles (eg, in connection with biographical ruptures). ${ }^{43-45}$

\section{TRHIV women's access to and retention in HIV care}

To analyse TRHIV women's access to and retention in HIV care, individual factors will be identified, including social factors (employment, living conditions, etc) and psychosocial factors (self-esteem, mental health, etc). We will also document their experience of discrimination and perceived stigma, and estimate the burden of each of these factors on access and retention.
Structural data collected on HIV care units will allow us to complement the above analyses by evaluating structural effects on the different indicators highlighted above (eg, the specific context of a hospital; the HIV care unit's technical and human resources available).

We will first perform a factor analysis of all 53 HIV care units to identify different profiles. HIV care units with similar characteristics will be grouped together (eg, large urban centres vs small centres in large cities vs small centres in small cities).$^{46}$ After this, we will perform multilevel analyses to disentangle individual barriers to care access and retention from their structural counterparts.

\section{Sexual health}

The data collected will document sexualities according to TRHIV women's gender affirmation trajectories, risk taking (sexual or substance use) and relationship to prevention. We will measure the impact of each of these factors on their sexual health needs in order to propose comprehensive HIV strategies and interventions for gender affirmation.

\section{COVID-19 health crisis impact on TRHIV women}

We will describe the impact of the ongoing COVID-19 health crisis on the everyday lives of TRHIV women, specifically in terms of HIV medical care, sexuality, social precarity (eg, financial resources, housing), and mental health.

\section{Specific needs of TRHIV men}

A thematic content analysis ${ }^{47}$ of the individual qualitative interviews with TRHIV men will be performed using the software package $\mathrm{NVIVO}^{48}$ to categorise the themes which emerge. Similar themes will be coded, compared and combined. They will then be compared with the textual variables obtained from the whole TRHIV men sample to highlight problems specific to that population in terms of HIV care access retention.

\section{Study limitations}

The fact that we are recruiting only TRHIV patients followed in hospital HIV care units means that those followed in primary care (ie, non-hospital contexts) will be missed. However, as all TRHIV patients must officially go to a hospital care unit at least once a year, it is possible that some will be recruited. TRHIV who refuse to participate will also be missed. Moreover, some TRHIV will probably be missed because HIV care units may not identify all potentially eligible patients.

\section{ETHICS AND DISSEMINATION \\ Ethical aspects}

Trans \& HIV is being conducted in accordance with the ethical principles set out in the current revised version of the Declaration of Helsinki (64th General Assembly of the World Medical Association, Fortaleza, Brazil, October 2013). 
Version 3.0 of the study (dated 7 September 2020) involves the processing of personal data for the purposes of study, evaluation and research not involving humans. The study is officially recognised as being of public interest and complies with France's 004 reference methodology for simplified access to research data. It was approved by Inserm's Ethical Evaluation Committee (approval number: 20-694 on 12 May 2020) and is registered with the National Commission on Informatics and Liberty under the number 2518030720).

\section{Information, consent and data confidentiality}

Potential participants are informed about the study through the information note provided by the attending physician in each of the participating HIV care units. It is provided before any data collection. Patients are given time to reflect before deciding to participate or not. Each patient must be informed that their participation is voluntary and that they are free to withdraw from the study at any time without justification, and that their withdrawal will in no way have negative consequences on the quality of care their doctor will continue to provide. Answering the quantitative questionnaire (TRHIV women) or participating in the qualitative interview (TRHIV men) constitutes consent.

All the information collected on the study participants will remain strictly confidential and coded. No data will show the name, address, or any other participant information which would lead to their direct identification. Each participant is assigned an anonymous, six-character identifier code (number of the investigating centre, entry number of the person in the centre according to trial entry order) which is entered in all survey documents.

\section{Dissemination}

All the results from the ANRS Trans\&HIV study published in peer-reviewed journals will be disseminated to the HIV transgender community, institutional stakeholders and healthcare providers. We will use adapted materials, general public leaflets and articles in French-language journals to disseminate them.

The ANRS Trans\&HIV survey will provide information previously unavailable in France on the living conditions and life trajectories of TRHIV.

The areas explored will provide us with a greater understanding of the consequences of TRHIV life trajectories on the management of their disease (poor quality of life, loss of income, poor mental health). The discrimination experienced, in terms of the timing of participants' HIV infection in their life trajectory, may be useful to inform public policy and develop prevention strategies for the whole trans community (HIV positive or negative). The results of this research will allow us to better understand TRHIV health needs in order to suggest possible national recommendations for comprehensive HIV and transition medical care for this population.

\section{Author affiliations}

${ }^{1}$ Aix Marseille Univ, Inserm, IRD, ISSPAM, SESSTIM UMR 1252, Marseille, ProvenceAlpes-Côte d'Azur, France

${ }^{2}$ ORS PACA, Observatoire Régional de la Santé Provence-Alpes-Côte d'Azur, Marseille, Provence-Alpes-Côte d'Azur, France

${ }^{3}$ ACCEPTESS-T, Paris, France

${ }^{4}$ Service de médecine interne, UFR Paris Île-de-France Ouest, Hopital AmbroisePare, Boulogne-Billancourt, Île-de-France, France

${ }^{5}$ Inserm, IAME, UMR 1137, université Paris Diderot, Hopital Bichat - Claude-Bernard, Paris, Île-de-France, France

${ }^{6}$ Paris Descartes University, IRD, ERL Inserm SAGESUD, CEPED, Paris, Île-de-France, France

${ }^{7}$ Collectif Interassociatif coordination, Groupe Interassociatif Traitement \& Recherche Thérapeutique (TrT5), Paris, France

${ }^{8}$ AIDES, Pantin, Île-de-France, France

${ }^{9}$ Community-Based Research Laboratory, Coalition Internationale Sida PLUS, Pantin, France

Acknowledgements We thank all the HIV care units participating in ANRS Trans\&HIV despite the very complicated ongoing COVID-19 context; specifically, we would like to thank the heads of the units, doctors, and clinical study technicians who are helping us with the data collection. Special thanks to all the participating patients who are giving their valuable time to answer our questions and without whom this study could not progress. We also thank the associations ACEPTESS-T, AIDES, PASTT and the ANRS for its financial support. Finally, our thanks to Jude Sweeney (Milan, Italy) for the English translation and editing of our manuscript.

Contributors BS is the principal investigator and oversaw the study protocol development. MM, MB and GM contributed to the design of the research project. GR, DL, DM and TA contributed to the community involvement in the research project and helped construct the questionnaires. FM, YY, AFM, and ER drafted the medical questions to explore and helped with the selection of HIV care units. JP built the life-events questionnaire. MM wrote the first draft of the manuscript. All authors contributed to and approved the current version of the manuscript.

Funding ANRS Trans\&HIV is supported by the French National Institute for Health and Medical Research (Inserm) and French Agency for Research on AIDS and Viral Hepatitis (ANRS) - grant number ETCZ87324).

Competing interests None declared.

Patient consent for publication Not applicable.

Provenance and peer review Not commissioned; externally peer reviewed.

Supplemental material This content has been supplied by the author(s). It has not been vetted by BMJ Publishing Group Limited (BMJ) and may not have been peer-reviewed. Any opinions or recommendations discussed are solely those of the author(s) and are not endorsed by BMJ. BMJ disclaims all liability and responsibility arising from any reliance placed on the content. Where the content includes any translated material, BMJ does not warrant the accuracy and reliability of the translations (including but not limited to local regulations, clinical guidelines, terminology, drug names and drug dosages), and is not responsible for any error and/or omissions arising from translation and adaptation or otherwise.

Open access This is an open access article distributed in accordance with the Creative Commons Attribution Non Commercial (CC BY-NC 4.0) license, which permits others to distribute, remix, adapt, build upon this work non-commercially, and license their derivative works on different terms, provided the original work is properly cited, appropriate credit is given, any changes made indicated, and the use is non-commercial. See: http://creativecommons.org/licenses/by-nc/4.0/.

ORCID iD

Marion Mora http://orcid.org/0000-0001-7543-6982

\section{REFERENCES}

1 Chiam Z, Duffy S, Gil MG. Nigel Timothy Mpemba Pate. In: Trans legal mapping report 2019: recognition before the law. Genève: ILGA World, 2020.

2 Nolan IT, Kuhner CJ, Dy GW. Demographic and temporal trends in transgender identities and gender confirming surgery. Transl Androl Urol 2019;8:184-90.

3 Scheim Al, Bauer GR. Sex and gender diversity among transgender persons in Ontario, Canada: results from a respondent-driven sampling survey. J Sex Res 2015;52:1-14. 
4 Bauer G, Scheim A. Transgender people in Ontario. Canada: Statistics from the Trans PULSE Project to Inform Human Rights Policy, 2015.

5 Pilling MD. Invisible identity in the workplace: intersectional madness and processes of disclosure at work. Disabil Stud Q 2012;33.

6 Bauer GR, Zong X, Scheim Al, et al. Factors impacting transgender patients' discomfort with their family physicians: a RespondentDriven sampling survey. PLoS One 2015;10:e0145046.

7 Bauer GR, Pyne J, Francino MC, Caron Francino M, et al. Suicidality among trans people in Ontario: implications for social work and social justice. Serv Soc Que 2013;59:35-62.

8 Adams N, Hitomi M, Moody C. Varied reports of adult transgender suicidality: synthesizing and describing the peer-reviewed and gray literature. Transgend Health 2017;2:60-75.

9 Yüksel Sahika, Aslantas Ertekin B, Öztürk M, et al. A clinically neglected topic: risk of suicide in transgender individuals. Noro Psikiyatr Ars 2017;54:28-32.

10 Narang P, Sarai SK, Aldrin S, et al. Suicide among transgender and Gender-Nonconforming people. Prim Care Companion CNS Disord 2018;20. doi:10.4088/PCC.18nr02273. [Epub ahead of print: 21 Jun 2018].

11 Longman Marcellin R, R. Bauer G, I. Scheim A. Intersecting impacts of transphobia and racism on HIV risk among trans persons of colour in Ontario, Canada. Ethnicity and Inequalities HSC 2013;6:97-107.

12 Becasen JS, Denard CL, Mullins MM, et al. Estimating the prevalence of HIV and sexual behaviors among the US transgender population: a systematic review and meta-analysis, 2006-2017. Am J Public Health 2019;109:e1-8.

13 Mayo-Wilson L, Benotsch E, Grigsby S. Combined effects of gender affirmation and economic hardship on vulnerability to HIV: a qualitative analysis among U.S. adult transgender women. BMC Public Health 2020;20.

14 Sevelius JM, Carrico A, Johnson MO. Antiretroviral therapy adherence among transgender women living with HIV. J Assoc Nurses AIDS Care 2010;21:256-64.

15 Baral SD, Poteat T, Strömdahl S, et al. Worldwide burden of HIV in transgender women: a systematic review and meta-analysis. Lancet Infect Dis 2013;13:214-22.

16 Poteat T, Scheim A, Xavier J, et al. Global epidemiology of HIV infection and related Syndemics affecting transgender people. $J$ Acquir Immune Defic Syndr 2016;72 Suppl 3:S210-9.

17 Mizuno Y, Frazier EL, Huang P, et al. Characteristics of transgender women living with HIV receiving medical care in the United States. LGBT Health 2015;2:228-34.

18 Dowshen N, Matone M, Luan X, et al. Behavioral and health outcomes for HIV+ young transgender women (YTW) linked to and engaged in medical care. LGBT Health 2016;3:162-7.

19 Radix A, Sevelius J, Deutsch MB. Transgender women, hormonal therapy and HIV treatment: a comprehensive review of the literature and recommendations for best practices. J Int AIDS Soc 2016;19:20810.

20 Pommier J-D, Laouénan C, Michard F, et al. Metabolic syndrome and endocrine status in HIV-infected transwomen. AIDS 2019;33:855-65.

21 Lacombe-Duncan A, Bauer GR, Logie CH, et al. The HIV care cascade among transgender women with HIV in Canada: a mixedmethods study. AIDS Patient Care STDS 2019;33:308-22.

22 Clements-Nolle K, Marx R, Guzman R, et al. Hiv prevalence, risk behaviors, health care use, and mental health status of transgender persons: implications for public health intervention. Am J Public Health 2001;91:915-21.

23 Reisner SL, White JM, Mayer KH, et al. Sexual risk behaviors and psychosocial health concerns of female-to-male transgender men screening for STDs at an urban community health center. AIDS Care 2014;26:857-64.

24 Scheim Al, Bauer GR, Travers R. Hiv-Related sexual risk among transgender men who are gay, bisexual, or have sex with men. $J$ Acquir Immune Defic Syndr 2017;74:e89-96.

25 LOI n 2016-1547 Du 18 novembre 2016 de modernisation de la justice Du XXle siècle (1) 2016.

26 Haute Autorité de Santé (HAS). Rapport HAS 2009.pdf, 2009.

27 Broussy S, Reigadas S, Vandenhende M. Caractéristiques sociodémographiques, identifications de genre, parcours de transition médicopsychologiques et $\mathrm{VIH} /$ sida dans la population trans. Premiers résultats d'une enquête menée en France en
2010, 2011. Available: /paper/Caracte\%CC\%81ristiquessociode \%CC\%81mographiques\%2C-de-genre\%2C-Broussy-Reiga das/0be043481378139c368123d687868dafcacfd116 [Accessed 16 Dec 2020].

28 Giami A. Caractéristiques sociodémographiques, identifications de genre, parcours de transition médicopsychologiques et $\mathrm{VIH} / \mathrm{sida}$ dans la population trans. In: Premiers résultats d'une enquête menée en France en. 12, 2010.

29 Biello KB, Hughto JMW. Measuring intersectional stigma among racially and ethnically diverse transgender women: challenges and opportunities. Am J Public Health 2021;111:344-6.

30 D'Almeida Wilson K, LERT F, Berdougo F. Kaygan d'Almeida Wilson. pdf, 2008.

31 LERT F, Annequin M, Tron L. Situation socioéconomique des personnes vivant avec le VIH suivies l'hôpital en France métropolitaine en 2011. Premiers résultats de l'enquête ANRS-Vespa2, 2013. Available: /maladies-et-traumatismes/ infections-sexuellement-transmissibles/vih-sida/situationsocioeconomique-des-personnes-vivant-avec-le-vih-suivies-a-lhopital-en-france-metropolitaine-en-2011.-premiers-resultats-de-Ienquete-an [Accessed 7 Jun 2021].

32 Marsicano E, Dray-Spira R, Lert F, et al. Multiple discriminations experienced by people living with HIV in France: results from the ANRS-Vespa2 study. AIDS Care 2014;26 Suppl 1:S97-106.

33 Bail HL, Giametta C, Rassouw N. Que pensent les travailleur.se.s a Du sexe de la loi prostitution. 101, 2016.

34 Lyons T, Krüsi A, Pierre L, et al. Negotiating violence in the context of Transphobia and Criminalization: the experiences of trans sex workers in Vancouver, Canada. Qual Health Res 2017;27:182-190.

35 Raho-Moussa M, Guiguet M, Michaud C, et al. Respective roles of migration and social deprivation for virological non-suppression in HIV-infected adults on antiretroviral therapy in France. PLoS One 2019;14:e0213019.

36 Bouscarat F, Pommier J-D, Michard F, et al. Infection à VIH, infections sexuellement transmises et complications dermatologiques chez les sujets transgenres consultant en centre hospitalo-universitaire. Résultats préliminaires chez 399 patients. Annales de Dermatologie et de Vénéréologie 2015;142:S485.

37 Bertin C, Abbas R, Andrieu V, et al. Illicit massive silicone injections always induce chronic and definitive silicone blood diffusion with dermatologic complications. Medicine 2019;98:e14143.

38 Leung MW, Yen IH, Minkler M. Community based participatory research: a promising approach for increasing epidemiology's relevance in the 21st century. Int J Epidemiol 2004;33:499-506.

39 Katz-Wise SL, Pullen Sansfaçon A, Bogart LM, et al. Lessons from a community-based participatory research study with transgender and gender nonconforming youth and their families. Action Res 2019;17:186-207.

40 Beauchemin C, Hamel C, Lesné M. Discrimination: a question of visible minorities, 2010.

41 Desgrées du Loû A, Parcours LF. Parcours de vie et santé des Africains immigrés en France. Paris: La Découverte, 2017. https:// www.cairn.info/parcours-de-vie-et-sante-des-africains-immigres9782707196453.htm

42 Le Cœur S, Im-Em W, Koetsawang S. Vulnérabilité et vie avec le VIH en Thaïlande : l'apport de l'approche biographique. Population 2005;60:551-68.

43 JONES BL, NAGIN DS, ROEDER K. A SAS procedure based on mixture models for estimating developmental trajectories. Sociol Methods Res 2001;29:374-93

44 Nagin DS, Tremblay RE. Analyzing developmental trajectories of distinct but related behaviors: a group-based method. Psychol Methods 2001;6:18-34.

45 Nagin DS, Odgers CL. Group-Based trajectory modeling (nearly) two decades later. J Quant Criminol 2010;26:445-53.

46 Coulaud P-J, Protopopescu C, Ndiaye K, et al. Individual and healthcare supply-related barriers to treatment initiation in HIVpositive patients enrolled in the Cameroonian antiretroviral treatment access programme. Health Policy Plan 2021;36:137-48.

47 Paillé P, Mucchielli A. Chapitre 11 - L'analyse thématique, 2012: 231-314.

48 Association pour la recherche qualitative. Introduction l'analyse qualitative informatisée l'aide du logiciel QSR Nvivo 2.0. Rimouski: $\mathrm{ARQ}, 2005$ 